\title{
Bony Ankylosis of the Subtalar Joint in Gout: A case report
}

\author{
by S.M. Shareef ${ }^{1}$, S. Sinha ${ }^{2} \otimes$, A.C. Campbell ${ }^{3}$
}

The Foot \& Ankle Journal 1 (11): 2

Bony ankylosis is a rare consequence of gouty arthritis. Ankylosis is typically a complication of long standing arthritis. Literature reports have shown that anti-hyperuricemic agents can reverse the urate deposition in a joint, but has no effect on the progression of eventual anklyosis of a joint. In this case, gouty ankylosis of the subtalar joint is reported after a relatively short episode of gout despite the use of anti-hyperuricemic agents and NSAID therapy.

Key words: Gout, anti-hyperuricemic agents, ankylosis, subtalar joint

Published: November, 2008

This is an Open Access article distributed under the terms of the Creative Commons Attribution License. It permits unrestricted use, distribution, and reproduction in any medium, provided the original work is properly cited. @The Foot \& Ankle Journal (www.faoj.org)

Bony ankylosis in gout is extremely rare. The few cases that have been reported in literature typically occur in patients with chronic, suboptimally treated or severe gout. We report a case of bony ankylosis in a patient with gouty arthritis of short duration.

\section{Case Report}

A 43-year-old female presented with pain and swelling of the left ankle of four-month duration. There was no previous history of trauma, infection, or any other joint problem. Physical examination revealed fullness over the medial aspect of the ankle with mild tenderness. There was some restriction of movements of the ankle and subtalar joints. Laboratory tests revealed a normal ESR and CRP with Rheumatoid factor, Anti-nuclear antibody and HLA B27 antigen being negative. Serum uric acid was elevated.

Address correspondence to: S. Sinha. Department of Orthopaedics Monklands, Hospital. Monkscourt Avenue. Airdrie ML6-OJS United Kingdom

1,2,3 Department of Orthopaedic Surgery, Monklands Hospital, Airdrie, UK.
Skin and nails did not show any psoriatic features. Radiographs of the ankle showed minor degenerative changes with slight narrowing of the subtalar joint space.

Patient was started on colchicine as an initial management, and subsequently commenced on allopurinol and NSAID's. In spite of these measures pain and swelling of the ankle worsened. A bone scan showed increased activity within the subtalar joint. Debilitating nature of symptoms, even with optimal medical treatment, warranted fusion of the subtalar joint. Synovial biopsy revealed a patchy chronic inflammatory cell infiltrate, but monosodium urate crystals were not detected.

At one-year follow up, the joint remained solidly fused both clinically and radiologically, and the symptoms had resolved completely. However, the patient complained of pain in the opposite ankle. Examination revealed pain with restriction of movement of the right subtalar joint. Patient was advised to continue with allopurinol and NSAID. 

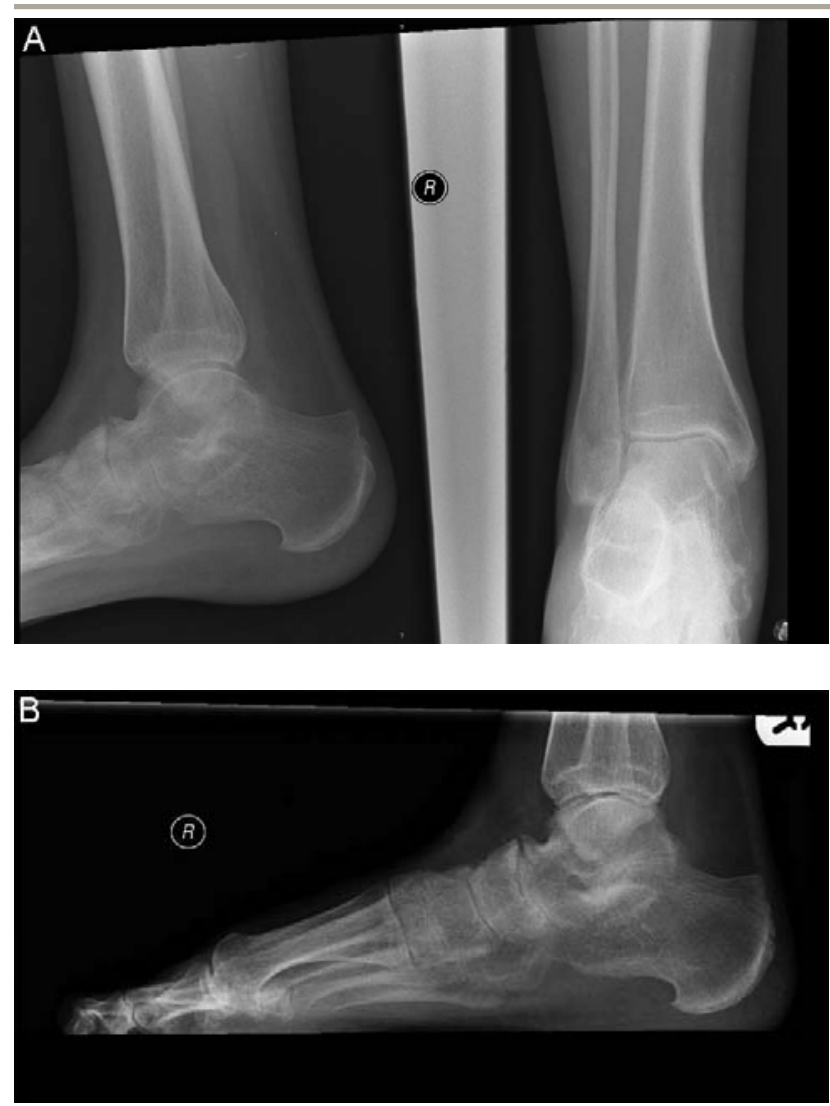

Figures 1 A and B AP and lateral radiograph of the right ankle showing ankylosis of the subtalar joint. (A) Lateral radiograph clearly showing the ankylosed subtalar joint. (B)

At review three months later, the right ankle pain had ceased. Radiographs revealed spontaneous ankylosis of the right subtalar joint without any intervention. (Figs. $1 \mathrm{~A}$ and B)

\section{Discussion}

Bony ankylosis is exceptional in gout patients. The first case was reported by Virchow in 1868 . Most reported gout patients with ankylosis had severe disease with onset during adolescence or early adulthood. ${ }^{1,2}$ Another common feature was suboptimal medical treatment. The most common sites of ankylosis were the carpus, tarsus and ankles. $^{3}$
A highly unusual feature in our case was the fact that the patient developed ankylosis within a short duration of the first acute attack on that side. The patient had no evidence of rheumatic disease known to cause ankylosis, such as ankylosing spondylitis, psoriatic arthritis, or diffuse idiopathic skeletal hyperostosis.

Although biopsy did not reveal gouty crystals, Pascual, et al., ${ }^{4}$ have shown total disappearance of crystals from joints in patients who were treated with urate lowering drugs, and have also determined the time needed for this to happen.

The pathophysiology of bony ankylosis in gout is not known. Anti-hyperuricemic agents can reverse urate deposition but may have no effect on the progression of ankylosis. ${ }^{5,6}$

\section{References}

1. Hughes GR, Barnes CG, Mason RM. Bony ankylosis in gout. Ann Rheum Dis. 27 (1): 67-70, 1968.

2. Ludwig AO, Bennet GA, Bauer W. A rare manifestation of gout: widespread ankylosis stimulating rheumatoid arthritis. Ann Intern Med. 11: 1248-1276, 1938.

3. Good AE, Rapp R. Bony ankylosis. A rare manifestation of gout. J Rheumatol. 5(3): 335- 337, 1978.

4. Pascual E, Sivera E. Time required for disappearance of urate crystals from synovial fluid after successful

hypouricaemic treatment relates to the duration of gout.

Ann Rheum Dis. 66: 1056-1058, 2007.

5. Cortet B, Duqesnoy B, Amoura I, Bourgeois P,

Delcambre B. Gout with ankylosis. Rev Rheum [Engl. Ed.]. 61(1): 44-47, 1994.

6. Shoji A, Yamanaka H, Kamatani N. A retrospective study of the relationship between serum urate level and recurrent attacks of gouty arthritis: evidence for reduction of recurrent gouty arthritis with antihyperuricemic therapy. Arthritis Rheum. 51 (3): 321-325, 2004. 